\title{
Nano-hydroxyapatite and its applications in preventive, restorative and regenerative dentistry: a review of literature
}

\author{
Erlind Pepla, MD \\ Lait Kostantinos Besherat, MD \\ Gaspare Palaia, DDS \\ Gianluca Tenore, DDS \\ Guido Migliau, DDS
}

Department of Oral and Maxillo-Facial Sciences, "Sapienza" University of Rome, Italy

Corresponding author:

Erlind Pepla

Department of Oral and Maxillo-Facial Sciences

"Sapienza" University of Rome

Viale Regina Elena 278/a

00198 Rome, Italy

E-mail: kledion2004@libero.it

\section{Summary}

This study aims to critically summarize the literature about nano-hydroxyapatite. The purpose of this work is to analyze the benefits of using nanohydroxyapatite in dentistry, especially for its preventive, restorative and regenerative applications. We also provide an overview of new dental materials, still experimental, which contain the nano-hydroxyapatite in its nano-crystalline form. Hydroxyapatite is one of the most studied biomaterials in the medical field for its proven biocompatibility and for being the main constituent of the mineral part of bone and teeth. In terms of restorative and preventive dentistry, nano-hydroxyapatite has significant remineralizing effects on initial enamel lesions, certainly superior to conventional fluoride, and good results on the sensitivity of the teeth. The nano-HA has also been used as an additive material, in order to improve already existing and widely used dental materials, in the restorative field (experimental addition to conventional glass ionomer cements, that has led to significant improvements in their mechanical properties). Because of its unique properties, such as the ability to chemically bond to bone, to not induce toxicity or inflammation and to stimulate bone growth through a direct action on osteoblasts, nano-HA has been widely used in periodontology and in oral and maxillofacial surgery. Its use in oral implantology, however, is a widely used practice established for years, as this substance has excellent osteoinductive capacity and improves bone-to-implant integration.
Key words: nano-HA, preventive, restorative, regenerative.

\section{Introduction}

The purpose of this work is to analyze what, up to now, is reported in the literature about the advantages of nano-hydroxyapatite in dentistry, especially in preventive and restorative dentistry, up to its use in oral surgery, such as implantology and periodontal regeneration. We also attempt to provide a broad overview of the new materials that are being born from experimental research, with particular attention to the materials commonly used in restorative dentistry, such as composite resins.

Tooth enamel is the most mineralized tissue of human body. Its composition is 96 wt.\% inorganic material and 4 wt. \% organic material and water. In dentin, the inorganic material represents $70 \mathrm{wt}$ \%. This inorganic material is mainly composed by a calcium phosphate related to the hexagonal hydroxyapatite, whose chemical formula is $\mathrm{Ca}_{10}\left(\mathrm{PO}_{4}\right)_{6} \cdot 2(\mathrm{OH})$. X-ray energy dispersive spectroscopy (EDS) analysis of enamel and dentin also indicated the presence in small quantities of other elements such as $\mathrm{Na}, \mathrm{Cl}$ and $\mathrm{Mg}$.

Hydroxyapatite (HA) is the main component of enamel, which gives an appearance of bright white and eliminates the diffuse reflectivity of light by closing the small pores of the enamel surface. Hydroxyapatite has long been among the most studied biomaterials in the medical field for both its proven biocompatibility and for being the main constituent of the mineral part of bone and teeth. Hydroxyapatite is also an important source of calcium and phosphate, very important for the remineralization of demineralized enamel areas. The inorganic component of all the mineralized tissues of the human body is, in fact, made up of a large prevalence of calcium phosphatesalts. Other inorganic materials such as calcium carbonates and sulphates are present in smaller quantities too; in particular hydroxyapatite represents $60-70 \%$ and $90 \%$ in weight of bone and enamel respectively. The recently developed interest for nanotechnology in many fields, is producing interesting and imminent applications in dentistry for nano-hydroxyapatite, which presents crystals ranging in size between 50 and $1000 \mathrm{~nm}$. The nano-hydroxyapatite has a strong ability to bond with proteins, as well as with fragments of plaque and bacteria, when contained in toothpastes. This ability is due to the size of nanoparticles, which considerably increase the surface area to which proteins can bind. Besides, nano- 
hydroxyapatite also acts as filler because it repairs small holes and depressions on enamel surface, a function enhanced by the small size of the particles that compose it. The Japanese company Sangi Co. Ltd was the first to take an interest in hydroxyapatite, after purchasing the rights ifrom NASA (U.S. National Aeronautics and Space Authority) in 1970. The astronauts, in fact, lost minerals from the teeth and bones in the absence of gravity, and NASA proposed a synthetic hydroxyapatite as a repairing material. The Sangi Co. Ltd had the idea in 1978 to launch toothpaste that could repair the tooth enamel, which contains for the first time nano-hydroxyapatite (Apadent). In 2006, the first toothpaste containing synthetic hydroxyapatite biomimetic as an alternative to fluoride for the remineralization and repair of tooth enamel appeared in Europe. The biomimetic hydroxyapatite function is to protect the teeth with the creation of a new layer of synthetic enamel around the tooth, rather than hardening the existing layer with fluorine, that chemically changes into calcium halophosphate $\left[\mathrm{Ca}_{5}\left(\mathrm{PO}_{4}\right)_{3} \mathrm{~F}\right]$.

In its granular form, hydroxyapatite is currently used in clinical dental practice to reconstruct periodontal bone defects, to the fill bone defects after cystectomy, after apicoectomy, after the loss of dental implants and to increase of the thickness of atrophic alveolar ridges. Shaped blocks of hydroxyapatite are especially used in maxillofacial surgery (bone defects after trauma, osteotomies and reductive stabilization, reconstruction of facial skeleton, replacement of parts of orbital and maxillary bone). Blocks, as well as granular powder, can also be used in pre-prosthetic surgery to increase the thickness of the alveolar ridge.

Studies on biocompatibility have shown that hydroxyapatite chemically binds to bone and induces no phenomena of toxicity nor inflammatory, local or systemic. Some researches show that the hydroxyapatite, unlike tricalcium phosphate, doesn't undergo resorption. Other authors have instead found resorption of hydroxyapatite. Thanks to its chemical and crystallographic affinity with inorganic components that constitute the bone, hydroxyapatite is able to establish chemical bonds and to ensure a more rapid integration of titanium implants to bone and surrounding tissues. Numerous studies have highlighted the role of hydroxyapatite in facilitating the process of osteointegration with or without other polymeric space.

\section{Analysis of the scientific literature (in restora- tive dentistry)}

The use of nano-hydroxyapatite as a material that could improve the properties of materials currently used in restorative dentistry has been studied. Moshaverina et al. in 2008 (1) have focused on the addition of $\mathrm{N}$-vinylpyrrolidone containing acids, nanohydroxyapatite and fluorapatite to conventional glass ionomer cements (GIC). These cements have unique properties such as biocompatibility, anticariogenic action (due to the release of fluorides) and adhesion to many dental structures. In this study the attention was paid to the search for materials to be added to common glass ionomer cements available on the market, Fuji II GC, in order to improve its mechanical properties.

Nano-hydroxy and fluorapatite have been synthesized using a sol-gel technique in an ethanol base.

The results showed that after 1 and 7 days, the nano$\mathrm{HA}$ /fluorapatite added to cements howed greater hardness to compression (CS) 177-179 MPa, a higher hardness to diametrical tension (DTS) 19-20 MPa and a higher hardness to biaxial flexibility (BFS) 26$28 \mathrm{MPa}$, compared to the control group (160 MPa in CS, 14 MPain DTS and $18 \mathrm{MPa}$ in BFS) (Tab. 1). Therefore, glass ionomers containing nano-bioceramics are very promising restorative dental materials with improved mechanical properties and strong binding to dentin, and may very soon replace GIC currently on the market. From these studies it seems to emerge, in fact, an unmistakable statistical datum: modified GICs with the above listed substances possess much higher capacity than traditional materials. Moshaverina et al. (2008) (2), have extensively studied the effects of incorporating hydroxyapatite and fluorapatite (FA) in a conventional glass ionomer cement (Fuji II GC). The addition of synthesized nano$\mathrm{HA}$ and FA in Fuji II improves the mechanical properties (to compressive, diametral tensile and biaxial flexural forces) of the resulting cement and its bonding strength to dentin. These bioceramics are, therefore, considered promising additives for glass ionomer cements used as restorative materials. However, perhaps due to the low solubility value of FA, the FA-containing samples showed very high values, after 7 and 30 days, in both mechanical properties and binding tests, as compared to compounds containing $\mathrm{HA}$ and to GIC.

In recent years, attention has focused towards the synthesis of new compounds of nano-HA. This is the case in the study on the remineralizing effects of zinc carbonate nano-HA $\left(\mathrm{ZnCO}_{3} / \mathrm{n}-\mathrm{HAP}\right)$ performed by Tschoppe et al. in 2011 (3). In the research, 35 bovine incisors were taken; from these teeth, 70 experimental blocks of enamel and 85 samples of dentin were obtained. A quarter of all the samples were coated with a special acid resistant paint, in order to act as control group. Enamel lesions were obtained by dipping the blocks in a solution (5I) containing $6 \mathrm{pM}$ of MHDP, $3 \mathrm{mMCa} \mathrm{Cl}_{2}$ dihydrate, $3 \mathrm{mM} \mathrm{K \textrm {K } _ { 2 }}$ $\mathrm{PO}_{4}$ and $50 \mathrm{mM}$ acetic acid, at a pH of 4.95, in an incubator ( $37^{\circ} \mathrm{C}$, BR 6000; Heraeus Kulzer) for 14 days. The lesions in dentin were prepared by dipping

Table 1. The synthesized nano-ceramic particles were incorporated in a powder of commercial glass ionomer (Fuji II GIC).

\begin{tabular}{lll}
\hline & Control Group & Nano-HA/Fluoroapatite Group \\
\hline CS & $160 \mathrm{MPa}$ & $177-179 \mathrm{MPa}$ \\
DTS & $14 \mathrm{MPa}$ & $19-20 \mathrm{MPa}$ \\
BFS & $18 \mathrm{MPa}$ & $26-28 \mathrm{MPa}$ \\
\hline
\end{tabular}


the samples in a solution containing $0.0476 \mathrm{mMNaF}$, $2.2 \mathrm{mMCaCl}_{2}$ dihydrate, $2.2 \mathrm{mM} \mathrm{KH}_{2} \mathrm{PO}_{4}, 50 \mathrm{mM}$ acetic acid and $10 \mathrm{mM} \mathrm{KOH}$, at a pH of $5.0\left(37^{\circ} \mathrm{C}\right)$ for five days. The $\mathrm{pH}$ value of the demineralizing solutions was constantly monitored. Afterwards, half of all demineralized surfaces were again covered with paint (baseline control demineralization). The samples were divided randomly into five groups (enamel $\mathrm{n}=14$, dentin $\mathrm{n}=17$ ) and were placed separately in remineralizing solution for two and for five weeks. In agreement with EN ISO 11609 standards, the respective toothpastes were diluted in a $1: 3$ ratio in the remineralizing solution, in order to obtain a homogeneous substance. Commercially available toothpastes containing $\mathrm{ZnCO}_{3} / \mathrm{n}-\mathrm{HA}$ or $\mathrm{n}-\mathrm{HA}$ (all without fluorides) were used while the toothpaste containing amino fluorides was used for the control group. Then, the samples were manually brushed with a soft brush and with a minimum pressure; this procedure was performed every day for about 5 seconds and with a contact time with the solutions of 115 seconds, a total time of 120 seconds. We must, however, emphasize that this procedure has some significant limitations as it is highly operator dependent and difficult to standardize and empirically assessable. After each brushing, the samples were rinsed with deionized water for 10 seconds. Every two days for each group, the solutions were changed $(250 \mathrm{ml})$. Finally, sections of 100 $\mathrm{mm}$ were performed and analyzed by means of microradiography and through an appropriate software (TMR for Windows 2.0.27.2; Inspektor Research System, Amsterdam, The Netherlands). Thirty samples of enamel and two of dentin were lost during preparation procedures. This complex in vitro study shows that toothpastes containing different types of nanohydroxyapatite have the same remineralizing capabilities on enamel and dentin, and those containing fluoride have lower capacity than the first. We must, however, take into account the limits of an in vitro study, as it is far from simulating the conditions present in the oral cavity.

In the study by Huang et al. of 2009 (4), the authors analyzed the remineralizing effect of nano-HA on demineralized bovine enamel under cyclical conditions of $\mathrm{pH}$, by the microhardness test, on cross-sections $(\mathrm{CSMH})$ and on surfaces and through polarized light microscopy (PLM). Nano-HA and conventional HA (crystals in the order of micrometers, from 0.5 to $2 \mu \mathrm{m}$ ), were obtained by the National Incubation Base of Nano-Biomaterials Industrialization, Sichuan University. The demineralizing solution (DS) used to create lesions similar to caries had the following composition: acetic acid50 mM, $\mathrm{Ca}\left(\mathrm{NO}_{3}\right)_{2} 2.2 \mathrm{mM}, \mathrm{KH}_{2} \mathrm{PO}_{4}$ $2.2 \mathrm{mM}$ and $\mathrm{NaF} 5.3 \mu \mathrm{M}$. The $\mathrm{pH}$ value of the $\mathrm{DS}^{2}$ was adjusted to 4.5 by the addition of a solution of $\mathrm{KOH}$. The remineralizing solution (RS) used under conditions of cyclical $\mathrm{pH}$ contains instead: HEPES $20 \mathrm{mM}$, $\mathrm{CaCl}_{2} 1.5 \mathrm{mM}, \mathrm{KH}_{2} \mathrm{PO}_{4} 0.9 \mathrm{mM}, \mathrm{KCl} 130 \mathrm{mM}$ and $\mathrm{NaN}_{3} 1 \mathrm{mM}$. The $\mathrm{pH}$ value was adjusted to 7.0 with $\mathrm{KOH}$. The dissolution of HA products was studied in preliminary experiments. In the study, incisors of 4 years old bulls were analyzed. The teeth, cut into blocks, were dipped in $8 \mathrm{ml}$ of DS for 72 hours at $37^{\circ}$ C. Finally, 70 teeth with a KHN (Knoop Hardness Number) value between 171.6 and 204.3 were selected. They were then divided into 10 groups exposed to different $\mathrm{pH}$ values. Afterward, the blocks were longitudinally sectioned in order to be studied by CSMH. The data were analyzed using SPSS 13.0 software. The remineralizing effect of nano-HA increased significantly when the $\mathrm{pH}$ was lower than 7.0.

One of the most important variables present in the mouth is the variation of $\mathrm{pH}$. The assessment of this variable is missing in this study. In another study by Huang et al. of 2009 (5), they analyzed the mineralizing ability of nano-hydroxyapatite in cyclically variable pH conditions.

The high availability of calcium and phosphate in these conditions, causes, according to the author, a positive effect on the remineralization of lesions. This indicates that nano-HA is a better resource of free$\mathrm{Ca}$, and this is important for the defense from dental caries and erosion. The largest increase in mineralization was observed in the group with $\mathrm{pH}$ 4.0. The group with $\mathrm{pH} 7.0$, however, showed the lowest degree of mineralization. An accumulation of many minerals in the lesions and a corresponding reduction of their depth were also observed. The effect of nano$\mathrm{HA}$ is better than the effect of micro-HA at $\mathrm{pH} 7.0$ and at the same concentrations. The concentration of calcium in solutions containing nano-HA was greater than that detected in solutions containing micro-HA. The $\mathrm{Ca}$ concentration increase leads to a growth in the saturation of oral fluids with HA, favoring the deposition of apatite minerals in the lesions and eventually promoting remineralization.

In terms of dental erosion, it is important to emphasize that its prevalence is increasing in young children and adolescents in developed and in developing countries. The main external cause of increased erosion is a higher consumption of acids in the diet and with drinks. In particular the use of sport drinks has recently increased, and these may cause erosion according to their acid content. With reference to this, is the interesting Min et al. in 2011 study (6), on nanohydroxyapatite as an addition to sports drinks. In this study was examined the possible beneficial effects of additioning nano-hydroxyapatite to sports drinks. Powerade ${ }^{\circledR}$ (PA) was taken as experimental solution and citric acid was added as acid. They prepared different solutions with the PA alone and with the addition of $0.05 \%, 0.10 \%$ and $0.25 \%$ nano-hydroxyapatite. 20 bovine teeth per group, cut in $3.5 \mathrm{~mm} \times 3.5$ $\mathrm{mm}$ blocks, were treated for 20 minutes three times a day, with $2 \mathrm{~h}$ and 40 min of interval between each treatment. Once the treatment process was finished, the samples were thoroughly rinsed with distilled water. Throughout the rest of the day, when not being treated, the teeth were immersed in a solution containing artificial saliva with the following composition: gastric mucin $0.22 \%, \mathrm{KCl} 14.93 \mathrm{mM}, \mathrm{KH}_{2} \mathrm{PO}_{4} 5.42$ $\mathrm{mM} \mathrm{NaCl} 6.51 \mathrm{mM}$ and $\mathrm{CaCl}_{2}$ dihydrate $1.45 \mathrm{mM}$. 
The cyclic $\mathrm{pH}$ process was repeated for 7 days. The potential erosion was determined by changes in the surface microhardness (SMH), and the teeth were analyzed with the confocal laser scanning microscopy (CLSM) and with the scanning electron microscope (SEM). The prevention of dental erosions increased with the concentration of n-HA, and sports drinks containing $0.25 \%$ of the substance have obtained the best results.

The consumption of carbonic acid containing drinks is the main etiological factor for tooth erosion. An experimental study on 18 permanent teeth dental erosion caused by beer was conducted by Hangoo et al. (11) in 2011 (7). These elements were subsequently treated with a remineralizing substance nano-hydroxyapatite based. In the study they primarily measured the microhardness of 18 permanent teeth (Fig. 1). Then they did second measurement of the teeth, after dipping them in a solution containing $40 \mathrm{ml}$ of beer (Behnoush Lemon Delester, Iran) for 5 minutes. The time was calculated according to actual studies on the permanence of beer in the mouth based on the amount drunk daily. The average microhardness primary values - i.e. prior to any type of manipulation of the teeth - , of the 18 cases was $340.24 \pm 25.4^{2}$ $\mathrm{kgf} / \mathrm{mm}$. This value reduced to $314.67 \pm 33.89^{2}$ (second value of microhardness) after immersion in beer; this is equivalent to $92.5 \%$ of the primary value of microhardness, and the "t" test analysis shows that this is statistically significant $(p=6.20)$. The value of secondary microhardness of the 9 cases in water was $312.85 \pm 36.79^{2} \mathrm{kgf} / \mathrm{mm}$; that reduces to $310.81 \pm$ $31.44^{2}$ (tertiary value of microhardness) after immersion in drinkable water. This is equivalent to $99.3 \%$, a value that is not statistically significant $(p=20.6)$. The secondary value of microhardness of the 9 cases dipped in NHAP was $315.18 \pm 30.65 \mathrm{~kg} / \mathrm{mm}$. This increases to $320.99 \pm 24.74^{2} \mathrm{kgf} / \mathrm{mm}$ (tertiary value of microhardness) after immersion in a solution with NHAP. This value is equivalent to $98.2 \%$, which is statistically significant $(p=0.012)$.

The results of this study demonstrate that there is a statistically significant increase in the microhardness of teeth demineralized by beer and then exposed to a solution of $\mathrm{n}-\mathrm{HA}$.

The aim of the study of Orsini et al. (8) is to evaluate the relative abilities of three desensitizing dentifrices to

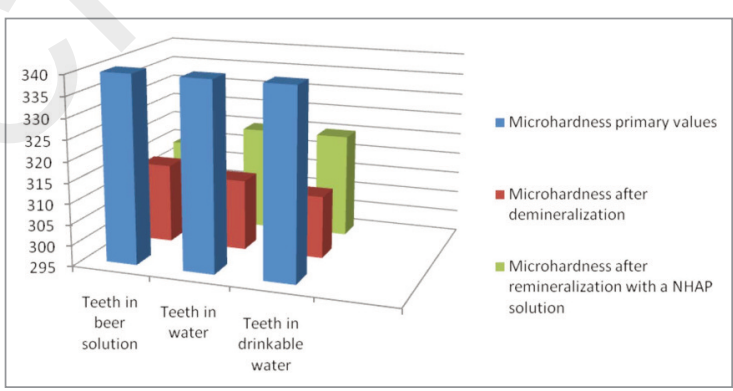

Figure 1. Microhardness values after demineralization and remineralization. provide rapid relief of dentin hypersensitivity $(\mathrm{DH})$. Using a double-mask, randomized design, three dentifrices: 1) containing $8 \%$ arginine and $1,450 \mathrm{ppm}$ sodium monofluorophosphate; 2) containing $8 \%$ strontium acetate and 1,040 ppm sodium fluoride; and 3) containing $30 \%$ microaggregation of zinc-carbonate hydroxyapatite nanocrystals were compared after 3-day treatment. Participant's DH was evaluated at baseline and after 3 days using air-blast, tactile, cold water, and subjective tests. The final sample consisted of 85 individuals: 29 received the arginine-based dentifrice (group 1), 27 the strontium acetate-based dentifrice (group 2), and 29 the dentifrice based on zinc-carbonate hydroxyapatite (group 3). All dentifrices were mostly effective to reduce $\mathrm{DH}$ : the percentage of score reduction from baseline to 3 days was $>30 \%$ for all tests (except for subjective test of group 2). The comparison among the three dentifrices showed that, after 3 days, there was an improvement in air-blast (mean percentage of reduction, $39.2 \%$ in group $1,42.0 \%$ in group 2, and $39.2 \%$ in group 3$)$, cold water $(41.5,51.8$, and $50 \%)$, tactile $(50.3,40.1$, and $33.8 \%)$, and subjective (33.1, 17.4, and $31.4 \%$ ) test scores, with differences being significant for cold water and subjective tests. For air-blast and tactile tests, there were no significant differences across groups at 3 days. Moreover, no significant differences at any test were observed in a subset of patients that were followed up to 8 weeks: all dentifrices were all highly efficacious. This study documents that the three tested dentifrices significantly reduced $\mathrm{DH}$ after 3 -day treatment, supporting their use in clinical practice. To the best of the authors' knowledge, this is the first report documenting the rapid relief from $\mathrm{DH}$ of a zinc-carbonate hydroxyapatite dentifrice. The study by Browing et al. of 2011 (9), finally focused attention on the search for a material that could reduce tooth sensitivity after bleaching. For this purpose, nano-hydroxyapatite was tested. It was noted that the teeth sensitivity after bleaching increased in the presence of enamel defects. Using a randomized clinical trial, the efficacy of a paste containing n-HA in reducing this type of sensitivity was analyzed. A paste containing n-HA (Renamel AfterBleach, Sangi Co. Ltd, Tokyo, Japan) and a placebo (zero-HA) were randomly assigned to 42 participants. A $7 \%$ hydrogen peroxide gel was used for 14 days, in association with a desensitizing paste used immediately for the 5 minutes afterwards. A diary was completed daily to note the effect of desensitization and the eventual sensitivity, on a VAS (Visual Analog Scale). Three aspects of the sensitivity of the teeth were analyzed: percentage of participants, number of days and intensity level. Color change was evaluated. For zero-HA and n-HAgroups, respectively, 51 and $29 \%$ of participants reported dentinal sensitivity $(p=0.06)$ (Tab. 2$)$. The days of sensitivity were 76 and 36 respectively $(p=0.001)$. The changes in VAS score from baseline have an upward trend in the zero-HA group ( $p=0.16)$ (Tab. 3). The color change was equivalent for both groups. The conclusions showed that the group treated with $\mathrm{n}-\mathrm{HA}$ had lower sensitivity levels. 
Table 2. Number (percentage) of participants with sensitivity to any assessment.

\begin{tabular}{llll}
\hline Number of assessments & Group & $\begin{array}{l}\text { Participants } \\
\text { with sensitivity }\end{array}$ & $\begin{array}{l}\text { Participants } \\
\text { without sensitivity }\end{array}$ \\
\hline Baseline & Zero-HA & $3(14 \%)$ & $18(86 \%)$ \\
First week whitening & Zero-HA & $14(67 \%)$ & $7(33 \%)$ \\
Second week whitening & Zero-HA & $7(33 \%)$ & $13(65 \%)$ \\
First week after whitening & Zero-HA & $5(25 \%)$ & $15(75 \%)$ \\
Baseline & n-HA & $2(10 \%)$ & $19(90 \%)$ \\
First week whitening & n-HA & $8(38 \%)$ & $13(62 \%)$ \\
Second week whitening & n-HA & $4(19 \%)$ & $17(81 \%)$ \\
First week after whitening & n-HA & $4(19 \%)$ & $17(81 \%)$ \\
\hline
\end{tabular}

$\mathrm{n}-\mathrm{HA}=$ nano-sizedhydroxyapatite, zero-HA=placebo

There is no significant difference between the groups during bleaching active phase (chi-square, $p=0.06$ )

Table 3. Percentage of days associated with sensitivity.

\begin{tabular}{llll}
\hline Number ofassessments & Group & Days with sensitivity & Days without feeling \\
\hline Baseline & Zero-HA & $9(6 \%)$ & $138(94 \%)$ \\
First week whitening & Zero-HA & $50(34 \%)$ & $97(66 \%)$ \\
Second week whitening & Zero-HA & $26(19 \%)$ & $114(81 \%)$ \\
First week after whitening & Zero-HA & $16(11 \%)$ & $124(89 \%)$ \\
Baseline & n-HA & $7(5 \%)$ & $140(95 \%)$ \\
First week whitening & n-HA & $20(14 \%)$ & $127(86 \%)$ \\
Second week whitening & n-HA & $16(11 \%)$ & $131(89 \%)$ \\
First week after whitening & n-HA & $14(10 \%)$ & $133(90 \%)$ \\
\hline
\end{tabular}

$\mathrm{n}-\mathrm{HA}=$ nano-sized hydroxyapatite, zero-HA=placebo

Participants in the group with $\mathrm{n}-\mathrm{HA}$ experience significantly more days with sensitivity during the active phase of bleaching (chi-square, $p=0.001$ )

\section{Analysis of the scientific literature (in oral surgery)}

Many researchers have focused on the use of nano$\mathrm{HA}$ as a co-adjuvant material in oral surgery, especially regarding the improvement of the dental implant characteristics. In the work of Masahiro et al. of 2012 (10), they analyze a new compound with nano-polymorphic crystalline HA applied to microrough titanium surfaces through a combination of flame spray and calcination at a low temperature. It was then analyzed for the biological capacity to increase bone-implant integration. The sandblasted microrough titanium implants and the titanium implants linked to HA and sandblasted were analyzed via biomechanical histomorphometric methods in rats.

In the study the HA used as an addition to implants surface is $55 \%$ crystalline and causes an increase of the surface area by $70 \%$ when compared to uncoated microrough surfaces. Furthermore, hydroxyapatite is free of impurities, with a calcium/phosphate ratio of 1.66 , thus being equivalent to the stoichiometric value of HA. Titanium cylinders $(1 \mathrm{~mm}$ in diameter and $2 \mathrm{~mm}$ long) were obtained from Grade 5 titanium alloy (Ti-6Al-4V). In order to create the micro-roughness, titanium samples were sandblasted with aluminum oxidemicro-powder. The HA coating was obtained by flame spray on blasted surfaces. The flame was created by means of acetylene and oxygen, and the air was used as a high-speed carrier. All examined surfaces were inspected by scanning electron microscope (SEM) (XL30; Philips, Eindhoven, The Netherlands) and by atomic force microscope (AFM) (SPM-9500J3; Shimadzu Corporation, Tokyo, Japan). Male Sprague-Dawley rats, about eight weeks old, were used for this study. The implant sites were prepared at about $10 \mathrm{~mm}$ from the distal edge of the femur using a $0.8 \mathrm{~mm}$ round bur and widening by means of reamers (\# ISO 090 and 100). A cylindrical implant that had been machined and sandblasted, or sandblasted and coated with HA was inserted into each hole prepared on the femur. The muscles and skin were then sutured separately with absorbable suture. The total number of animals used was78 (54 animals for testing machined implants, sandblasted, sandblasted and coated with $\mathrm{HA}$; analyzed at 2, 4 and 8 weeks; 24 animals for histological analysis for the groups of sandblasted and for sandblasted and coated with HA; analyzed at 2 and 4 weeks). HA-coated implants showed an increased percentage of bone-implant contact and an increase in bone volume within $50 \mu \mathrm{m}$ close to the implant surface. On the contrary, around implants coated with $\mathrm{HA}$, the bone volume outside the boundary of 50 $\mu \mathrm{m}$ was low. In particular, this study demonstrated that nano-crystalline hydroxyapatite is indisputably effective in increasing osteoconductivity and inhibiting the infiltration of soft tissue around the implant, but the effect is quite limited to the microenvironment around the implant.

Ceramic nanoparticles represent an encouraging class of bone graft substitutes due to their improved 
osteointegrative properties. Nano-crystalline hydroxyapatite binds bone and stimulates bone healing encouraging osteoblastic activity. In the study conducted by Singh et al. in 2012 (11), the authors examine the clinical and radiographic results obtained with NCHA bone graft (Sybograf ${ }^{\circledR}$ ) associated with collagen membranes (Periocol ${ }^{\circledR}$ ), compared with OFD (Open Flap Debridement) in the treatment of intrabony periodontal defects.

In the work on the comparison between NCHA associated bone grafts with collagen membranes and traditional OFD technique, Singh et al. (11) designed a controlled randomized clinical trial in parallel groups. Eighteen intrabony defects in 14 patients aged between 25 and 65 years were randomly assigned to a test group and to a control group. The plaque index, the gingival index, the PPD (probing pocket depth), the clinical attachment level (CAL) and the gingival recession (REC) were recorded at baseline, and were reassessed 6 months later (Tab. 4). Furthermore, bone grafts were evaluated through digital software. In the test sites they have placed NCHA bone graft with collagen membranes, while in control sites they performed only OFD. Recall appointments were set at 7 days, 30 days, and then at 3 months and 6 months (Tab. 4). The association between resorbable membranes, derived from fish, and NCHA bone grafts is particularly positive as concern to the improvement of periodontal indices. This work has clearly shown that the additional use of resorbable membranes derived from fish in combination with NCHA bone grafts is clinically, radiographically and statistically significant compared to OFD alone, in terms of reduction of PPD, CAL gain and percentage of bone filling.

In the study by Qu et al. in 2010 (12), the use of a bioactive and osteoconductive composite formed by nano-hydroxyapatite and polyamide 66 (nHA/PA66) was tested, for the creation of a new asymmetric porous membrane to be used for guided bone regeneration (GBR). Regarding the membranes, they analyzed the cytotoxicity of the material, the response of the surfaces to bone formation, the morphology, proliferation and cell cycle progression of bone marrow stromal cells (BMSCs) in rat culture. The polygonal and fusiform shape of BMSCs was observed using a scanning electron microscope (SEM). In the research the PA66 with an average viscosity, molecular weight

Table 4. The plaque index, the gingival index, the PPD (probing pocket depth), the clinical attachment level (CAL) and the gingival recession (REC).

\begin{tabular}{lll}
\hline & Control group & Test group \\
\hline PPD reduction & $3.22 \pm 1.09 \mathrm{~mm}$ & $4.33 \pm 0.5 \mathrm{~mm}$ \\
& $p=0.007$ & $p=0.007$ \\
CAL gain & $2.77 \pm 1.09 \mathrm{~mm}$ & $3.77 \pm 0.66 \mathrm{~mm}$ \\
& $p=0.006$ & $p=0.006$ \\
REC increase & $0.55 \pm 0.72 \mathrm{~mm}$ & $0.49 \pm 0.52 \mathrm{~mm}$ \\
& $p=0.025$ & $p=0.046$ \\
Main gain in filled & $2.07 \pm 0.67 \mathrm{~mm}$ & $0.91 \pm 0.21 \mathrm{~mm}$ \\
radiografic defect & $p=0.007$ & $p=0.008$ \\
\hline
\end{tabular}

(Mv) 18 k Dawas used, derived from BASF (Ludwigshafen, Germany). The suspension of nano-hydroxyapatite used for the compound was prepared with a wet method and hydrothermal treatments. The proliferation of BMSCs culture on nHA/PA66 membranes has been tested with the MTT method (MTT: [3 - \{4,5-dimethylthiazol-2YL\}-2,5-diphenyl-2H-tetrazoliumbromide]), and it was found to be higher than the negative control group after 1 and 4 days of incubation and, moreover, did not appear to have any significant differences after 7 and 11 days of culture. The results of the cell cycle suggest that the membrane has no negative influence on cell division. The membrane has an asymmetric porous structure, in which pores smaller than 10 microns are distributed on one side (microporous layer), while pores ranging between 30 and $200 \mu \mathrm{M}$ are located on the opposite side (macroporous layer). The microporous layer of the membrane prevents the migration of fibrous connective tissue in bone defects, being also able to permeate sufficient nutrients for regenerating tissue. The results show that $n-H A / P A 66$ membrane is a $3 D$ porous structure with a dense microporous layer on one side and with a spongy microporous layer on the other side. In vitro experiments show that nHA/PA66 membrane has a good affinity for attaching to BMSCs, and a non-negative effect on cells viability and proliferation. The results of in vitro and in vivo studies indicate that the nHA/PA66 membrane has an excellent biocompatibility and is indicated for use in guided tissue regeneration (GTR) or GBR.

Recent studies suggest that nano-crystalline hydroxyapatite (nano-HA) paste represents a promising class of grafting bone substitutes. The study of Kasai et al. of 2008 (13) was conducted to investigate the proliferation of human periodontal ligament (PDL) in cell cultures, in the presence of a nano-HA paste, and to analyze cells' associated signal paths. In this experimental research on the consequences of the application of nano-HA in cell culture with human periodontal ligament (PDL), PDL cells were stimulated with pastes of nano-HA and with an enamel matrix derivative (EMD) in a soluble form. The proliferation of PDL cells was determined by analyzing the incorporation of bromodeoxyuridine (BrdU) in the DNA of proliferating cells. In order to understand the mechanism that underlies the increase in cell proliferation of PDL cells exposed to nano-HA, the phosphorylation of serine/threonine protein kinase Akt was analyzed using phospho-specific antibodies. The nanoHA paste showed a potential for proliferation, two times lower than EMD, but both substrates significantly increased the proliferation rate $(p<0.05)$ in comparison to the negative control group. In conclusion, it seems that the growth and proliferation rate of PDL cells in the presence of nano-HA paste is mechanically connected to the activation of the receptor for the epidermal growth factor receptor (EGFR) and its downstream targets ERK1/2 and Akt. In conclusion, these studies suggest that nano-HA paste is a potent stimulator of cell proliferation, which probably 
contributes to the fundamental process of periodontal tissue regeneration.

\section{Final remarks}

The nano-hydroxyapatite is a revolutionary material with a wide use in dentistry. With regard to restorative and preventive fields, nano-hydroxyapatite has remarkable remineralizing effects on initial lesions of enamel, certainly higher than traditional fluorides used until now for this purpose. Nano-hydroxyapatite is, in fact, a better source of free $\mathrm{Ca}$, and this is a key element as regards the remineralization, the protection against caries and dental erosion. With regard to the latter point, of fundamental importance in dentistry, the road leading to the addition of small percentages of nano-HA $(0.25 \%)$ in beverages such as mineral supplements for sports activities, in order to prevent tooth erosion caused by those drinks, seems very promising. Nano-HA has also been used as a supplementary material, in order to improve the dental materials already existing and widely used. This is the case of the experimental addition to traditional GIC, a procedure that has led to significant improvements in the mechanical properties of these substances.

A continuing interest in the nano-crystalline structure of hydroxyapatite has prompted many researchers to look for new combinations that could improve existing materials or create new ones that could meet their needs. This has led to new complex compounds, such as nano-HA associated with zinc carbonate, which seems to be an excellent material for the remineralization of initial lesions involving enamel and dentin, or as the nano-HA associated with the polyamide 66 , used in order to create a new periodontal membrane with improved properties.

Noteworthy are the applications of nano-HA in fields other than strictly restorative or preventive. Because of its unique properties, such as the ability to chemically bind to bone, without inducing toxicity or inflammation and stimulating bone growth through a direct action on osteoblasts, nano-HA has been widely used in periodontology and in oral and maxillofacial surgery. Collagen membranes associated with nano$\mathrm{HA}$ are used to fill bone defects, since this substance leads to a clear improvement in periodontal indices. Its use in the field of implantology, instead, is a practice widely used and has been consolidated in recent years, since the nano-hydroxyapatite has excellent osteoinductive capacity and improves osteointegration in bone-implant interface. It is therefore natural to expect for the coming years an increased interest in this revolutionary substance and a growing number of scientific articles on the subject.

\section{References}

1. Moshaverina A, Ansari S, Moshaverina M, Roohpour N, Darr J. A, Rehman I. Effect of incorporation of hydroxyapatite and fluoroapatitenanobioceramics into conventional glass ionomer cements (GIC). Acta Biomaterialia. 2008; Volume 4, Issue 2, March:432-440.

2. Moshaverina A, Ansari S, Movasaghi Z, Billington RV, Darr JA, Rehman IU. Modification of conventional glass-ionomer cements with $\mathrm{N}$-vynilpyrrolidone containing polyacids, nanohydroxy and fluoroapatite to improve mechanical properties. Dent Mater. 2008 Oct;24(10): 1381-90. Epub 2008 Apr 22.

3. Tschoppe P, Zandim DL, Martus P, Kielbassa AM. Enamel and dentin remineralization by nano-hydroxyapatite toothpastes. J Dent. 2011 Jun;39(6):430-7. Epub 2011 April 8.

4. Huang S, Gao S, Cheng L, Yu H. Remineralization potential of nano-hydroxyapatite on initial enamel lesion: an in vitro study. Caries Res. 2011;45(5):460-8. Epub 2011 Sep 2.

5. Huang SB, Gao SS, Yu HY. Effect of nano-hydroxyapatite concentration on remineralization of initial enamel lesion in vitro. Biomed Master. 2009 Jun 4;(3):034104. Epub 2009 Jun 5.

6. Min JH, Kwon HK, Kim BI. The addition of nano-sized hydroxyapatite to a sport drink to inhibit dental erosion: in vitro study using bovine enamel. J Dent. 2011 Sep,39(9):62935. doi: 10.1016/j.dent.2011.07.001. Epub 2011 Jul 7.

7. Hangoo R, Abbasi F, Rezvani MB. Evaluation of the effect of nanohydroxyapatite on erosive lesions of the enamel of permanent teeth following exposure to soft beer in vitro. Scientific Research and assays. 2011; vol 6 (26):5933-5936.

8. Orsini G, Procaccini M, Manzoli L, Sparabombe S, Triduzzi $\mathrm{P}$, Bambini F, Putignano A. A 3-day randomized clinical trial to investigate the desensitizing properties of three dentifricies. J Periodontal. 2013 Nov;84(11):e65-73. doi: 10.1902/jop.2013.120697. Epub 2013 Mar 14;

9. Browing WD, Sopanis DC, Deschepper EJ. Effect of nanohydroxyapatite Paste on Bleaching-related Tooth Sensitivity. Journal of Esthetic and restorative Dentistry. 2011.

10. Masahiro Y, Takeshi U, Naoki T, Takayuki I, Kaori N, Norio $\mathrm{H}$, Takeo S, Takahiro O. Bone integration capability of nanopoyimorphic crystalline hydroxyapatite coated on titanium implants. Int J Nanomedicine. 2012;7:859-873.

11. Singh VP, Nayak DG, Shah D. Clinical and radiographic evaluation of Nano-crystalline hydroxyapatite bone graft (Sybograft) in combination with bioresorbable collagen membrane (Periocol) in periodontal intrabony defects. Dent Res J (Isfahan). 2012 Jan;9(1):60-7.

12. Qu Y, Wang P, Man Y, Li Y, Zuo Y, Li J. Preliminary biocompatible evaluation of nano-hydroxyapatite/polyamide 66 composite porous membrane. Int J Nanomedicine. 2010 Aug 9;5:429-35.

13. Kasai A, Willershausen B, Reichert C, Rohring B, Smeets $R$, Schmidt M. Ability of nanocrystalline hydroxyapatite paste to promote human ligament cell proliferation. J Oral Sci. 2008 Sep;50(3):279-85. 